\title{
Establishment and Characterization of a Singaporean Chinese Lung Adenocarcinoma Cell Line with Four Copies of the Epidermal Growth Factor Receptor Gene
}

\author{
Meng Ling Choong, Jacklyn Yong, Yu Wang, and May Ann Lee
}

\begin{abstract}
We have established a lung adenocarcinoma cell line, ETCC016, from lung pleural effusion of a male Singaporean Chinese with advanced lung adenocarcinoma. The subject smoked 20 cigarettes per day for more than 30 years. The cell line arose from spontaneous transformation of cells grown in a collagen-coated culture dish. Transformed characteristics of the cell line include the ability to reach high confluency in a culture dish, low cell doubling time, ability to form colonies in soft agar, and ability to form solid tumor in immune-compromised SCID mice. Immunostaining showed that the cells originated from lung epithelial cells. Genomic analysis revealed a large amount of chromosomal aberrations (gain and loss of genetic materials, and loss of heterozygosity $[\mathrm{LOH}])$, indicative of a long history of smoking. The cells have four copies of epidermal growth factor receptor (EGFR) and three copies of $M Y C$, but have lost one copy of the RB1 gene. LOH was detected in TP53 and BRAF genes. There is no anaplastic lymphoma kinase $(A L K)$ gene rearrangement. The ETCC016 lung adenocarcinoma cell line has demonstrated susceptibility towards inhibitors specific for EGFR/HER2 and ALK targets, but resistance to MYC-specific inhibitor. This cell line will be a useful model for further understanding of lung adenocarcinoma.
\end{abstract}

Key words: adenocarcinoma; ALK; array CGH; chemosensitivity; EGFR; FISH; lung; mutation

\section{Introduction}

$\mathbf{L}$ UNG CANCER IS ONE OF THE LEADING CAUSES of cancerrelated death in Singapore and around the world. Between 2005 and 2009, about 1193 people were diagnosed with lung cancer yearly in Singapore. The great majority of them were smokers. The risk of lung cancer for a smoker is 15 to 25 times more than a nonsmoker. ${ }^{1}$ Recent data from the Singapore National Health Survey conducted in 2011 indicated an alarming increase in the number of Singaporeans smokers: $14.3 \%$ of adults are now smokers compared with $12.6 \%$ in 2004. Of even greater concern is the prevalence of smoking among young Singaporeans aged 18 to 29 years which has jumped to $16.3 \%$ from $12.3 \%$ in 2004 -this represents a $33 \%$ increase in just over 6 years. ${ }^{2}$ Smoking increases the risk of all major histologic types of lung cancer, a finding that was recognized in the 1980 US Surgeon General's Report. ${ }^{3}$

Lung cancer is a heterogeneous disease where diverse genetic and epigenetic abnormalities have been reported. ${ }^{4}$ Lung cancer has been traditionally classified into small cell lung cancer and non-small cell lung cancer (NSCLC) based on histological characteristics. NSCLC is further categorized into squamous cell carcinoma, large cell carcinoma, and adenocarcinoma. Lung adenocarcinomas account for approximately $40 \%$ of lung cancers. ${ }^{5}$ The molecular underpinnings of the different lung cancers have become increasingly clear recently. This has facilitated the development of targeted therapies with specific inhibitory drugs. ${ }^{6}$

Results from polymorphism and genome-wide association studies showed that Asians and Caucasians may have different genetic susceptibilities to lung cancer. Compared to Caucasian patients with NSCLC, East Asian patients have a much higher prevalence of epidermal growth factor receptor $(E G F R)$ mutation (approximately $30 \%$ vs. $7 \%$, predominantly among patients with adenocarcinoma and never-smokers), a lower prevalence of Kirsten rat sarcoma viral oncogene (KRAS) mutation (less than $10 \%$ vs. 18\%, predominantly among patients with adenocarcinoma and smokers), and higher proportion of patients who are responsive to EGFR tyrosine kinase inhibitors. ${ }^{7}$ A specific study in East Asia revealed that $90 \%$ of lung adenocarcinoma from never-smokers was found to harbor well-known oncogenic mutations in

Cell-Based Assay Development Group, Experimental Therapeutics Centre, Agency for Science Technology and Research, Singapore. 
just four genes (EGFR mutation, echinoderm microtubuleassociated protein-like 4 [EML4]-anaplastic lymphoma kinase $[A L K]$ fusion, receptor tyrosine-protein kinase erbB-2 [HER2] insertion, and KRAS mutation). Other mutations, including PIK3C mutations (always together with EGFR mutations) and TP53 mutations, ${ }^{8}$ were reported.

Using lung pleural effusion from a 53-year-old Singapore Chinese male smoker with advanced lung adenocarcinoma, we established a spontaneously transformed continuous cell line, ETCC016. Validation and authentication of the identity of this cell line have been carried out. The cell line has four copies of the EGFR gene and numerous other mutations. It also has the ability to engraft and form solids tumor rapidly in immune-compromised mice. The ETCC016 cell line will be a valuable tool for biomedical discovery and research in lung adenocarcinomas especially in the Chinese population.

\section{Materials and Methods}

\section{Cancer tissue and establishment of cell line}

Pleural effusion was obtained from a 53-year-old man of Singapore Chinese origin with advanced adenocarcinoma of the lung. The cells were grown on plates coated with collagen-1 (Life Technologies, Carlsbad, CA) in Dulbecco's modified Eagle's medium (DMEM) supplemented with $10 \%$ fetal bovine serum (FBS), L-glutamine, and penicillin/ streptomycin (Life Technologies). A colony of cells was transferred to plates without the collagen- 1 coating in the same culture medium. Cell cultures were maintained in a humidified incubator at $37^{\circ} \mathrm{C}$ with $5 \%$ atmospheric $\mathrm{CO}_{2}$.

\section{Cell line authentication and virology safety testing}

Cell pellets were sent to IDEXX Laboratories (Columbia, MO) for authentication and virological safety testing. The tests done were short tandem repeat (STR) analysis to establish cell line identity, polymerase chain reaction (PCR) to detect interspecies (rat, mouse, Chinese hamster, African Green Monkey) contamination, and PCR to screen for 19 types of virus and mycoplasma contamination.

\section{Immunofluorescence staining for cell-specific markers}

Primary antibodies for epithelial membrane antigen (EMA) (Dako, Glostrup, Denmark), vimentin (Abcam, Cambridge, MA), pan-cytokeratin (pan-CK) (Abcam), epithelial cell adhesion molecule (EpCAM) (Santa Cruz, Dallas, TX), lung epithelial uteroglobin-related protein 1 (UGRP1) (Santa Cruz), and caveolin-1 (Santa Cruz) were purchased. Secondary antibodies used were Alexa Fluor 594 goat anti-rabbit (Life Technologies) and Alexa Fluor 488 goat anti-mouse (Life Technologies). Antibody concentrations used in the immunofluorescence staining were as recommended by the antibody manufacturers.

\section{Cell population doubling time}

Doubling time for the cells was determined using the IncuCyte real-time cell analyzer (Essen Bioscience, Ann Arbor, MI). Cells $\left(1 \times 10^{5}\right)$ were seeded in T-25 flasks and placed in the IncuCyte. Cell growth was monitored until confluency was achieved. Analysis of cell growth was performed with the IncuCyte software.

\section{Comparative genomic hybridization}

Cell pellets containing $1 \times 10^{6}$ cells were sent to Origen Labs (Singapore) for comparative genomic hybridization $(\mathrm{CGH})$ array hybridization using the Affymetrix SNP 6.0 platform. Data analysis was performed with Affymetrix Chromosome Analysis Suite.

\section{ALK gene rearrangement study}

Cells in interface were probed using the Vysis ALK Break Apart Fluorescence In Situ Hybridization (FISH) Probe Kit (Abbott, DesPlaines, IL) according to the manufacturer's protocol by the Molecular Diagnosis Centre, National University Hospital, Singapore.

\section{Soft agar assay for anchorage independent cell growth}

Soft agar colony formation assay was done in 24-well plates. Each well contained $0.6 \mathrm{~mL}$ of $0.6 \%$ agar (Sigma, St. Louis, MO) in complete medium in the bottom layer, $0.5 \mathrm{~mL}$ of $0.36 \%$ agar in complete medium with cells in the middle layer, and covered with $0.5 \mathrm{~mL}$ medium. The cells were cultured at $37^{\circ} \mathrm{C}$ with $5 \%$ atmospheric $\mathrm{CO}_{2}$ for 2 to 3 weeks. After overnight staining with tetrazolium dye 3-(4,5-dimethylthiazol-2-yl)-2,5-diphenyltetrazolium bromide (MTT; Sigma) at $70 \mu \mathrm{L}$ per well at $5 \mathrm{mg} / \mathrm{mL}$, the colonies were counted using the GelCount ${ }^{\circledR}$ instrument (Oxford Optronix, Oxford, United Kingdom).

\section{Mouse tumorigenicity study}

The established cell line was injected subcutaneously into the right flank of eight female SCID mice (age 6-8 weeks) at 10 million cells per mouse. The animals were observed for clinical signs, body weight, tumor volume, and mortality. These parameters were recorded twice in a week throughout the experiment. The mice were sacrificed at the end of the experiment. A piece of each palpable tumor was snap frozen or fixed in $10 \%$ neutral-buffered formalin solution for histopathology study by hematoxylin and eosin staining. The study was carried out at the A*STAR Biological Resource Center (accredited by the Association for Assessment and Accreditation of Laboratory Animal Care). The study design was reviewed and approved by the Institutional Animal Ethics Committee.

\section{Sensitivity of cells towards a panel of specific kinase inhibitors}

Erlotinib, lapatinib, mubritinib, LDK378, AP26113, lonafarnib, and 10058-F4 were purchased from Selleck Chem (Houston, TX). Cells were seeded in $50 \mu \mathrm{L}$ of medium in 96-well plates at 8000 cells/well and incubated overnight. Compounds $(50 \mu \mathrm{L})$ were added to cells and incubated for $48 \mathrm{~h}$. Cell viability was measured using Cell TiterGlo (Promega, Madison, WI). Data was analyzed with GraphPad Prism software (La Jolla, $\mathrm{CA})$ and the half maximal inhibitory concentration $\left(\mathrm{IC}_{50}\right)$ was determined. Error bars denote standard deviation.

\section{Results}

\section{Spontaneous transformation and generation of the ETCC016 continuous cell line}

Cells were obtained from lung pleural effusion from a 53year-old male of Singapore Chinese origin with advanced 
adenocarcinoma of the lung. Cells from the pleural effusion were initially grown in a tissue culture dish coated with collagen- 1 to promote the attachment and proliferation of epithelial cells. ${ }^{9}$ Fresh culture medium was replaced every 4 days. However, most cells died after a couple of weeks and only a few cells remained attached on the dish. After about 2 months, a colony of tightly packed cells was observed (Fig. 1A). The cells were trypsinized and transferred to a new culture dish without collagen coating in DMEM supplemented only with $10 \%$ FBS. The cells were passaged every 4 days and they have been growing in culture for more than 100 passages. This continuous growing cell line is named ETCC016. Beside the ability to grow continuously, the cell line also demonstrated the ability to reach high cell density with short doubling time; both are characteristics of immortalized cells (Fig. 1B).

\section{Cell biomarkers}

Immunostaining was performed to determine the expression of specific cell markers (Fig. 1C). Epithelial cell markers pan-CK, EMA, and EpCAM were detectable in the cells. EMA is highly expressed by most adenocarcinoma and is associated with poor prognosis, ${ }^{10}$ while EpCAM is a membrane glycoprotein expressed in most normal human epithelium and in most carcinomas. ${ }^{11}$ Our cell line also expressed vimentin, a biomarker associated with epithelial cells that are involved in metastasis. ${ }^{12}$ UGRP1 is a secreted protein expressed in lung epithelial cells ${ }^{13}$ and caveolin- 1 is known to play a role in the development of NSCLCs. ${ }^{14}$ Collectively, the ex- pression of these markers indicated that our cell line is of lung epithelial cell origin.

\section{Authentication and virology safety testing}

ETCC016 was sent to IDEXX Laboratories for authentication and virology testing. The results showed that ETCC016 is of human origin and a unique DNA fingerprint profile of nine-allele STR markers was established (Table 1). There was no cross contamination from other animal species, and the cells were not infected with the 19 viruses and mycoplasma tested in the virology safety panel.

\section{Tumorigenicity study}

Anchorage-independent growth is another hallmark of cell transformation and is the gold standard used to detect transformed malignant cells in vitro. The ETCC016 lung adenocarcinoma cell line was able to form colonies in the soft agar assay (Fig. 2A), confirming its transformed nature. Furthermore, the cell line could engraft and form solid tumors in SCID mice. Complete acceptance rate (8/8) and rapid tumor growth were observed. Tumor volume data and mouse body weight are represented in Figure 2B. Histology analyses by hematoxylin and eosin staining revealed densely packed tumor cells forming glandular pattern consistent with adenocarcinoma. The tumor cells were poorly differentiated with fibrous stroma which was associated with invasive cancer cells (Fig. 2C). The tumor tissue was also stained positive for Ki-67 (a cellular marker for active proliferation), pan-CK,
A

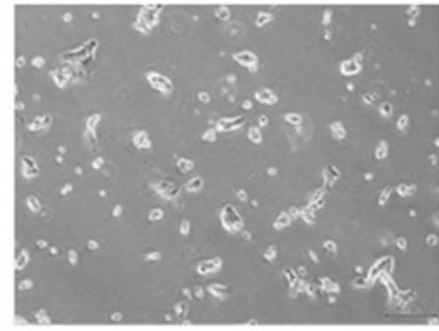

Collagen-1 coated plate, dormant for 2 months

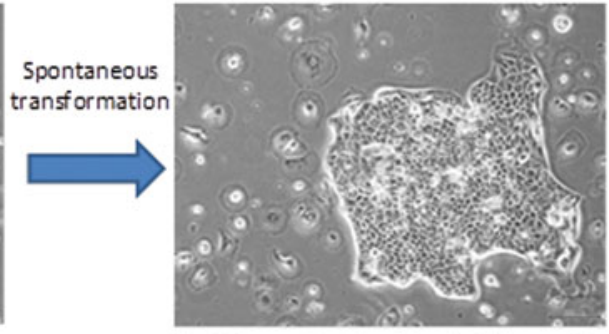

Tightly packed epithelial-like cells
B

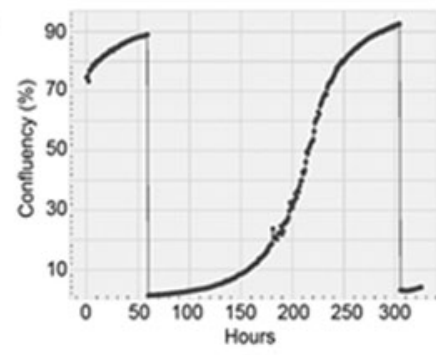

Cell doubling time $=24$ hours
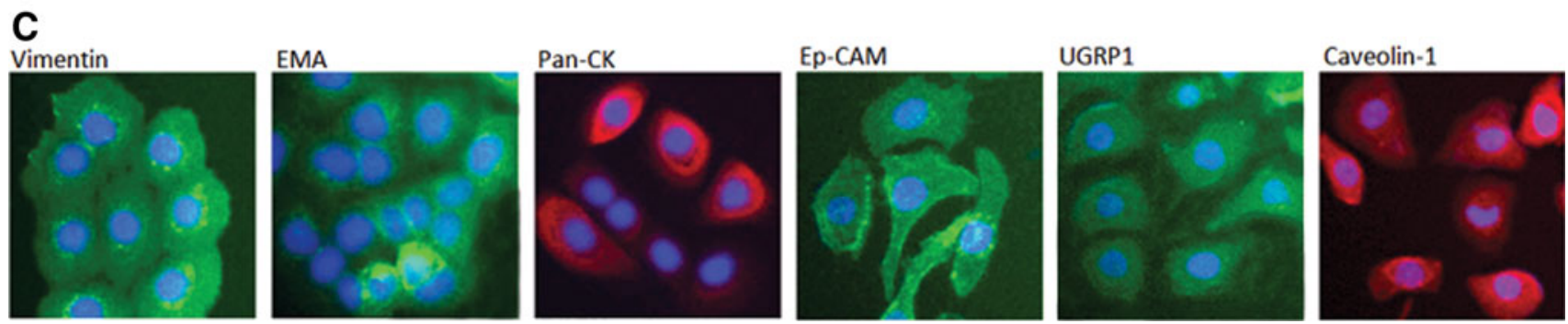

FIG. 1. Spontaneous transformation of cells from lung pleural effusion. (A) After being dormant for 2 months in collagen1-coated dish, tightly packed epithelial-like cell colonies were observed ( $\times 40$ magnification). (B) The cells grew to high confluency with a short cell doubling time, consistent with immortalized cells. (C) Immunostaining for cell markers. Epithelial cell markers EMA (green stain), pan-CK (red stain), and EpCAM (green stain) were detected. Vimentin (green stain), an epithelial-mesenchymal transition marker was also detected. Lung-specific markers UGRP1 (green stain) and caveolin-1 (red stain) were present. Nuclei were counter-stained blue with DAPI. Magnification: $\times 100$. EMA, epithelial membrane antigen; pan-CK, pan-cytokeratin; EpCAM, epithelial cell adhesion molecule; UGRP1, uteroglobin-related protein 1; DAPI, 4',6diamidino-2-phenylindole. 
Table 1. Authentication and Virological Safety Testing on ETCC016 Human Lung Adenocarcinoma CELl LiNe By IDEXX LABORATORIES

\begin{tabular}{|c|c|}
\hline & Alleles/results \\
\hline \multicolumn{2}{|l|}{ DNA fingerprinting $^{\mathrm{a}}$} \\
\hline Amelogenin & $\mathrm{X}$ \\
\hline CSF1PO & 10,12 \\
\hline D13S317 & 10 \\
\hline D16S539 & 12 \\
\hline D5S818 & 11,12 \\
\hline $\mathrm{D} 7 \mathrm{~S} 820$ & 9,12 \\
\hline TH01 & 9 \\
\hline TPOX & 8,11 \\
\hline vWA & 17,19 \\
\hline \multicolumn{2}{|l|}{ Species-specific PCR evaluation $^{\mathrm{b}}$} \\
\hline Mouse & - \\
\hline Rat & - \\
\hline Human & + \\
\hline African green monkey & - \\
\hline Chinese hamster & - \\
\hline \multicolumn{2}{|l|}{ Virology and mycoplasma safety ${ }^{c}$} \\
\hline Epstein-Barr virus & - \\
\hline Human adenoviruses & - \\
\hline Human cytomegalovirus & - \\
\hline Hepatitis A virus & - \\
\hline Hepatitis B virus & - \\
\hline Hepatitis $\mathrm{C}$ virus & - \\
\hline Human herpesvirus 6 & - \\
\hline Human immunodeficiency virus 1 & - \\
\hline Human immunodeficiency virus 2 & - \\
\hline Herpes simplex virus 1 & - \\
\hline Herpes simplex virus 2 & - \\
\hline Human T-lymphotropic virus 1 & - \\
\hline Human T-lymphotropic virus 2 & - \\
\hline Varicella zoster virus & - \\
\hline Hantaan virus & - \\
\hline Lymphocytic choriomeningitis virus & - \\
\hline Seoul virus & - \\
\hline Sin Nombre virus & - \\
\hline Mycoplasma sp. & - \\
\hline
\end{tabular}

${ }^{a}$ DNA fingerprinting was performed using nine unique STR markers. ${ }^{b}$ Species-specific PCR evaluation was carried out in five different animal species.

${ }^{\mathrm{c}}$ Virological and mycoplasma tests showed that the cell line is not contaminated with the 19 viruses and mycoplasma in the panel.

PCR, polymerase chain reaction; STR, short tandem repeat; +, present; -, absent.

and EMA, confirming that the tumor consisted of rapidly dividing epithelial cells.

\section{Karyotyping by comparative genomic hybridization}

Genomic analysis was performed to determine gross genomic changes that have occurred after cell transformation and immortalization. The CGH digital karyogram documented extensive genetic aberrations (Fig. 3). The amount of genetic changes in lung cancers is known to correlate to the quantity and duration of smoking. ${ }^{15}$ The ETCC016 cell line is derived from the lung adenocarcinoma of a chronic smoker who smoked about 20 cigarettes daily for more than 30 years. The chromosome X, chromosome 6q, 7, 8p, 11p, and 16q have extensive loss of heterozygosity ( $\mathrm{LOH})$. Chromosomes
$4 q, 18 q, 3 p, 8 p$, and $9 p$ and most of chromosome $2 q$ have a gain of genetic materials, while chromosomes $3 \mathrm{q}, 12 \mathrm{q}, 16 \mathrm{p}$, the lower arm of 8q, and most of 20q have a loss of genetic materials.

We then looked for driver mutations in lung adenocarcinomas. The common driver mutations are in the genes $E G F R$, HER2, ALK, and KRAS. ${ }^{16}$ Mutations in EGFR, HER2, KRAS, and $A L K$ are mutually exclusive in patients with NSCLC. The ETCC016 cell line has four copies of EGFR and no detectable chromosomal aberrations in HER2, ALK, or KRAS (Table 2). ALK was not found to have gene rearrangement by FISH (results not shown). Other molecular abnormalities in NSCLC are found in the oncogenes $M Y C$ and $B R A F$ and the tumor suppressor genes $R B 1$ and TP53. The cell line has three copies of the oncogene MYC and loss of one copy of the tumor suppressor gene $R B 1$, while LOH was detected in BRAF and TP53.

\section{Drug sensitivity of the cell line}

The ETCC016 lung adenocarcinoma cell line is found to be sensitive towards EGFR, HER2, and ALK specific inhibitors, with $\mathrm{IC}_{50}$ values in the submicromolar range (Table 3 ). This indicates that the growth of this cell line is dependent on the EGFR and ALK signaling pathways. EGFR and HER2 belong to the epidermal growth factor family, and compounds targeting EGFR and HER2 tend to cross-react due to structural homology between the two proteins. The ETCC016 cell line may harbor activating $A L K$ point mutations that were not detected in our CGH approach. Despite having three copies of $M Y C$, the cell line is not sensitive toward the MYC specific inhibitor 10058-F4.

\section{Discussion}

NSCLC is often insidious, and it may produce no symptoms until the disease is at an advanced stage. Early recognition of symptoms or biomarkers may be beneficial to improve the outcome. On initial diagnosis, $20 \%$ of patients have localized disease, $25 \%$ of patients have regional metastasis, and $55 \%$ of patients have distant spread of disease. ${ }^{17}$ Lung adenocarcinomas, the most common type of NSCLC, usually begin in tissues that lie near the outer part of the lungs, and they may be present for a long time before causing symptoms and being diagnosed. Lung adenocarcinoma is the most common form of lung cancer found in women, and they are often nonsmokers. It is also the most common type of lung cancer in people under the age of 45 and among all Asians. ${ }^{15}$ Cases of lung adenocarcinoma have been increasing in recent years, whereas another form of NSCLC, squamous cell carcinoma, has been decreasing. It is thought that the increase may be due to the addition of filters to cigarettes, which allow smoke to be inhaled more deeply into the lungs where adenocarcinoma occurs. ${ }^{18}$ The development of a Singapore Chinese lung adenocarcinoma cell line is timely and would contribute to better understanding of the molecular pathogenesis of this subset of NSCLC.

The ETCC016 lung adenocarcinoma cell line has four copies of EGFR. Activating EGFR mutations are more commonly observed in patients with lung adenocarcinomas with no history of smoking and in females of Asian descent. EGFR mutations are seen in approximately $30 \%$ of Asians and $7 \%$ of non-Asians, ${ }^{7}$ while another report showed that 
$\mathbf{A}_{\text {Softagar assay }}$

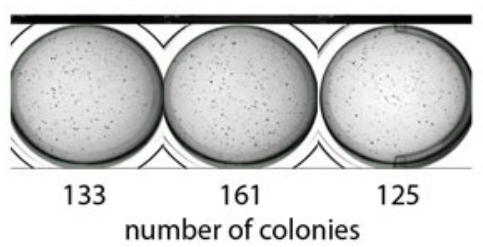

C Histology analyses of the tumor graft
B

Mouse tumorigenicity study ETCC016 tumor growth curve



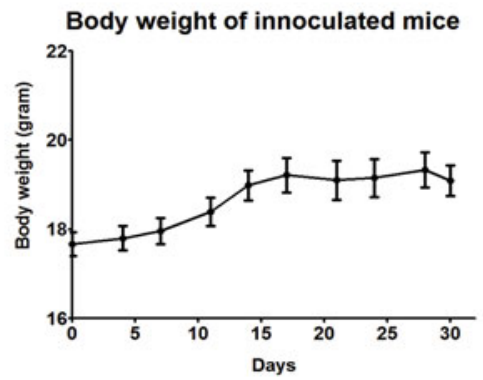

$H \& E$ staining

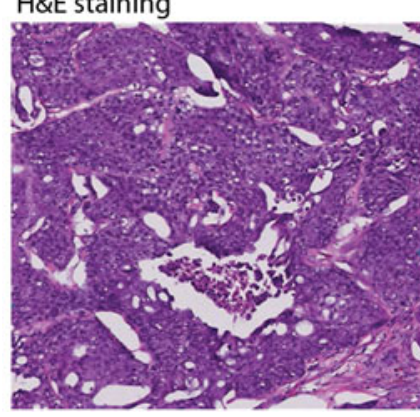

Ki-67 (brown)

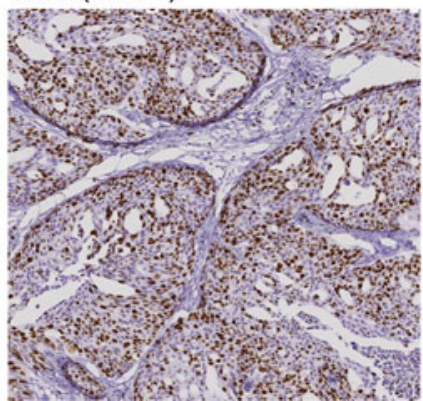

EMA (brown)

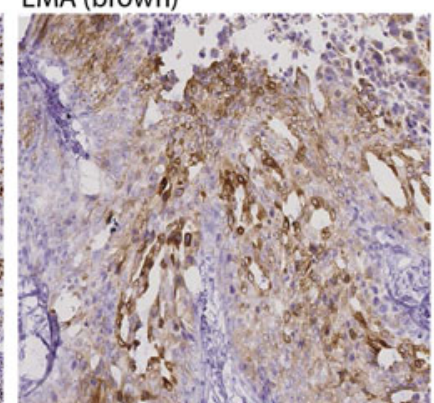

Pan-CK (brown)

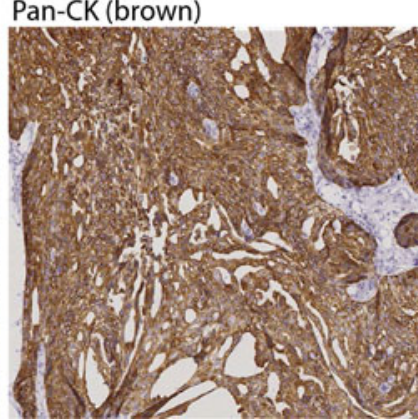

FIG. 2. Tumorigenicity study of the ETCC016 lung adenocarcinoma cell line by (A) in vitro soft agar assay and (B) in vivo tumor engraftment in SCID mice. Tumor volume and mouse body weight are shown. (C) Histology analyses of the tumor grafts are shown. The presence of Ki-67, EMA, and pan-CK are shown in brown in the histology slides. H\&E, hematoxylin and eosin.

about $80 \%$ of lung adenocarcinoma from East Asian neversmokers have EGFR mutations. ${ }^{8}$ EGFR tyrosine kinase inhibitors (EGFR TKIs) such as gefitinib, erlotinib, and afatinib are reversible competitive inhibitors of the tyrosine kinase domain of EGFR. Increased EGFR gene copy number, somatic activating mutations of the EGFR gene, and certain clinical and pathological features have been associated with dramatic tumor responses and favorable clinical outcomes with these agents in patients. ${ }^{19}$ The specific types of activat-

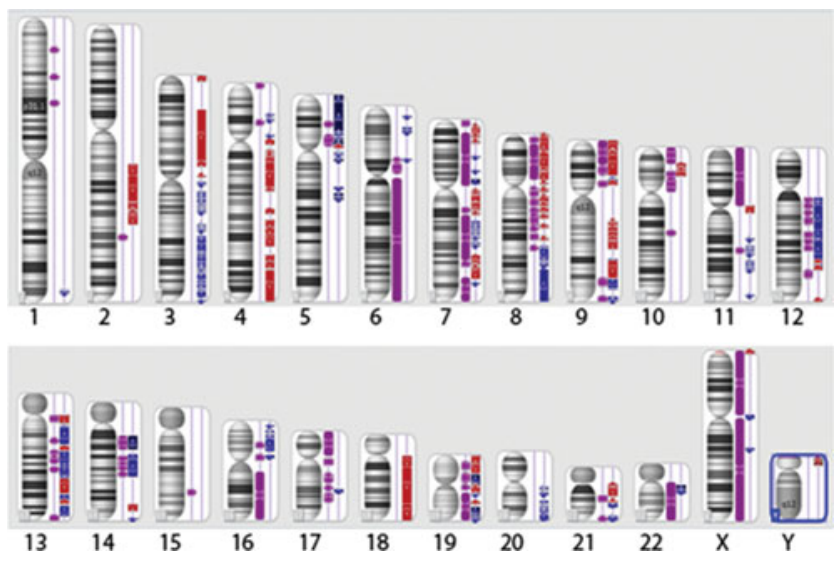

FIG. 3. Digital karyogram of the ETCC016 cell line showing large genetic aberrations. Gain of genetic materials is represented in red, loss of genetic materials is represented in blue, and $\mathrm{LOH}$ is represented in purple. $\mathrm{LOH}$, loss of heterozygosity. ing mutations that confer sensitivity to EGFR TKIs are present in the tyrosine kinase domain of the EGFR gene. The ETCC016 lung adenocarcinoma cell line is sensitive towards the EGFR inhibitor erlotinib $\left(\mathrm{IC}_{50}=0.19 \mu \mathrm{M}\right)$ and HER2 inhibitor mubritinib $\left(\mathrm{IC}_{50}=0.049 \mu \mathrm{M}\right)$.

Another common mutation found in lung adenocarcinoma is a small inversion within the short arm of chromosome 2 resulting in the fusion of the $\mathrm{N}$-terminal of the $E M L 4$ gene with the $A L K$ gene. There are many $E M L 4-A L K$ translocation variants and all have constitutive activation of the fusion proteins. ${ }^{4}$ We profiled the genetic changes in ETCC016 using array CGH. This method detects gain or loss in genetic materials and $\mathrm{LOH}$ but does not detect point mutation and translocation. We then determined that ETCC016 does not have $A L K$ gene rearrangement. Interestingly, ETCC016 is sensitive

Table 2. Reported Cancer Causal Genes in Lung ADENOCARCINOMAS ${ }^{\mathrm{a}}$

\begin{tabular}{lcr}
\hline Gene & Genetic changes & Chromosome \\
\hline EGFR & Gain $(+4)$ & $7 \mathrm{p} 11$ \\
HER2 (ERBB2) & NIL & $17 \mathrm{q} 12$ \\
ALK & NIL & $2 \mathrm{p} 23$ \\
KRAS & NIL & $12 \mathrm{p} 12$ \\
$M Y C$ & Gain $(+3)$ & $8 \mathrm{q} 24$ \\
$B R A F$ & LOH & $7 \mathrm{q} 34$ \\
$T P 53$ & LOH & $17 \mathrm{q} 13$ \\
$R B 1$ & Loss $(-1)$ & $13 \mathrm{q} 14$ \\
\hline
\end{tabular}

${ }^{\mathrm{a}} \mathrm{ETCC} 016$ was found to have four copies of EGFR and several other mutations. 
Table 3. Sensitivity of ETCC016 Towards a Panel of SPECIFIC Kinase InHIBITORS Response TO EGFR/HER2 AND ALK INHIBITORS

\begin{tabular}{lcl}
\hline Compound & $I C_{50}(\mu M)^{\mathrm{a}}$ & \multicolumn{1}{c}{ Class } \\
\hline Lapatinib & 1.62 & EGFR, HER2 inhibitor \\
Erlotinib & 0.19 & EGFR inhibitor \\
Mubritinib & 0.049 & HER2 inhibitor \\
AP26113 & 0.50 & ALK inhibitor \\
LDK378 & 0.76 & ALK inhibitor \\
Lonafarnib & 3.05 & KRAS inhibitor \\
10058-F4 & 69.4 & MYC inhibitor \\
\hline
\end{tabular}

${ }^{\mathrm{a}} \mathrm{IC}_{50}$ values at $48 \mathrm{~h}$ treatment are shown.

to two ALK inhibitors (AP26113 and LDK378 with $\mathrm{IC}_{50}$ at 0.50 and $0.76 \mu \mathrm{M}$, respectively). It could be that the ALK inhibitors have nonspecific targets, or the cells have ALK activating mutations that are undetectable using our array $\mathrm{CGH}$ and FISH approaches. Even though the EGFR mutations and EML4-ALK translocations are shown to be mutually exclusive in the same tumor, there are reports of patients with both mutations in rare cases. ${ }^{20}$

$K R A S$ mutations are also predominately found in lung adenocarcinomas and are seen in approximately $20 \%$ of cases in Caucasians but less than $10 \%$ in Asians. ${ }^{7}$ However, KRAS mutations are more common in smokers, with $16.5 \%$ detected in Chinese smokers. ${ }^{21}$ Patients with KRAS mutations seem to have a poorer prognosis and appear to be resistant to EGFR TKIs. ${ }^{22}$ Our array CGH results and the low drug sensitivity of the cells towards the KRAS inhibitor lonafarnib $\left(\mathrm{IC}_{50}=3.05 \mu \mathrm{M}\right)$ suggested that ETCC016 does not have KRAS mutation. On the other hand, despite having three copies of the MYC gene, the ETCC016 is not sensitive to the MYC inhibitor $10058-\mathrm{F} 4\left(\mathrm{IC}_{50}=69.4 \mu \mathrm{M}\right)$. This suggests that the extra copy of the $M Y C$ gene is either not active or not contributing to tumor growth. It is also possible that the survival of ETCC016 is more dependent on signaling through the EGFR and/or ALK pathway(s) than through the MYC pathway.

The ETCC016 is derived from cells harvested from lung pleural effusion. Unfortunately, we do not have access to the original lung tissue from which the cell line was derived to compare the genotypic and phenotypic properties with the lung cancer cell line. Nevertheless, a recent study has compared a variety of properties of 12 human NSCLC cell lines, cultured for a median period of 39 months, with their corresponding tumor tissues. ${ }^{23}$ The authors found that there is an excellent concordance between the lung tumor cell lines and their corresponding tumor tissues in the morphology $(100 \%)$, presence of aneuploidy $(100 \%)$, immunohistochemical expression of HER2/neu (100\%) and p53 proteins (100\%), LOH at 13 chromosomal regions analyzed $(97 \%)$, microsatellite alterations $(75 \%)$, and TP53 (67\%) and KRAS $(100 \%)$ gene mutations. The cell lines tend to show more aneuploidy and have higher incidences of TP53 mutations and microsatellite alterations. This is not surprising considering that more accumulated mutations are needed to immortalize the cells. Overall, NSCLC cell lines appeared representative of the lung cancer tumor from which they were derived and thus provide suitable model systems for biomedical studies of this important neoplasm. ${ }^{23,24}$
There are more than 200 reported lung cancer cell lines. ${ }^{24}$ More than 23 lung adenocarcinoma cell lines are available commercially from the American Type Culture Collection. However, all of them are of Caucasian origin, and lung cell lines of East Asian or Chinese origin are rare. There are recent publications announcing the development of five Chinese NSCLC cell lines with EGFR mutations ${ }^{25}$ and a lung adenocarcinoma cell line with bone metastases potency. ${ }^{26}$ As these cell lines with specific geographical associated genetic changes (such as EGFR mutations) are not freely available to scientists outside Asia, ${ }^{24}$ there is a need to establish more cell lines from Asian origin. The cell line established in our laboratory will be available to the research community and add to the limited number of available Asian lung adenocarcinoma cell lines. It would be an invaluable tool in the investigation of lung cancer biology and genetics.

\section{Acknowledgment}

We thank Chon Boon Eng from the National University Health System of Singapore (NUHS) Tissue Repository for providing the primary tissue used in this article.

\section{Author Disclosure Statement}

No competing financial interests exist.

\section{References}

1. National Cancer Centre Singapore. Lung cancer. Available at: www.nccs.com.sg/PatientCare/WhatisCancer/Typesof Cancer/Pages/lung-cancer.aspx p $=/$ PatientCare $\quad($ accessed February 24, 2014).

2. Picco L, Subramaniam M, Abdin E, et al. Smoking and nicotine dependence in Singapore: findings from a crosssectional epidemiological study. Ann Acad Med Singapore. 2012;41:325-334.

3. US Department of Health and Human Services. The Health Consequences of Smoking for Women: A Report of the Surgeon General. Public Health Service, Office of the Assistant Secretary for Health, Office on Smoking and Health: Washington, DC; pp. 109-131; 1980.

4. Luo S, Lam D. Oncogenic driver mutations in lung cancer. Tran Resp Med. 2013;1:6.

5. Travis WD, Travis LB, Devesa SS. Lung cancer. Cancer. 1995;75:191-202.

6. West L, Vidwans SJ, Campbell NP, et al. A novel classification of lung cancer into molecular subtypes. PLoS One. 2012;7:e31906.

7. Zhou W, Christiani DC. East meets West: ethnic differences in epidemiology and clinical behaviors of lung cancer between East Asians and Caucasians. Chin J Cancer. 2011; 30:287-292.

8. Sun Y, Ren Y, Fang Z, et al. Lung adenocarcinoma from East Asian never-smokers is a disease largely defined by targetable oncogenic mutant kinases. J Clin Oncol. 2010;28: 4616-4620.

9. Wu R. Growth of human lung tumor cells in culture. In: Culture of Human Tumor Cells. Pfragner R, Freshney RI (eds.) Wiley-Liss Inc.: Hoboken, NJ; pp. 1-21; 2004.

10. McGuckin MA, Walsh MD, Hohn BG, et al. Prognostic significance of MUC1 epithelial mucin expression in breast cancer. Human Pathol. 1995;26:432-439.

11. Trebak M, Begg G, Chong JM, et al. Oligomeric state of the colon carcinoma-associated glycoprotein GA733-2 
(Ep-CAM/EGP40) and its role in GA733-mediated homotypic cell-cell adhesion. J Biol Chem. 2001;276:2299-2309.

12. Leader M, Collins M, Patel J, et al. Vimentin: an evaluation of its role as a tumour marker. Histopathology. 1987; $11,63-72$.

13. Niimi T, Keck-Waggoner CL, Popescu NC, et al. UGRP1, a uteroglobin/Clara cell secretory protein-related protein, is a novel lung-enriched downstream target gene for the T/EBP/ NKX2.1 homeodomain transcription factor. Mol Endocrinol. 2001;15:2021-2036.

14. Sunaga N, Miyajima K, Suzuki M, et al. Different roles for caveolin-1 in the development of non-small cell lung cancer versus small cell lung cancer. Cancer Res. 2004;64:42774285 .

15. Kenfield SA, Wei EK, Stampfer MJ, et al. Comparison of aspects of smoking among the four histological types of lung cancer. Tobacco Control. 2008;17:198-204.

16. Zhou JX, Yang H, Deng Q, et al. Oncogenic driver mutations in patients with non-small-cell lung cancer at various clinical stages. Ann Oncol. 2013;24:1319-1325.

17. Lindeman NI, Cagie PT, Beasley MB, et al. Molecular testing guideline for selection of lung cancer patients for EGFR and ALK tyrosine kinase inhibitors: guideline from the College of American Pathologists, International Association for the Study of Lung Cancer, and Association for Molecular Pathology. J Thorac Oncol. 2013;8:823-859.

18. Marugame T, Sobue T, Nakayama T, et al. Filter cigarette smoking and lung cancer risk; a hospital-based case-control study in Japan. Br J Cancer. 2004;90:646-651.

19. Gazdar AF. Activating and resistance mutations of EGFR in non-small-cell lung cancer: role in clinical response to EGFR tyrosine kinase inhibitors. Oncogene. 2009;28(suppl 1): S24-31.

20. Zhang X, Zhang S, Yang X, et al. Fusion of EML4 and ALK is associated with development of lung adenocarcinomas lacking EGFR and KRAS mutations and is correlated with ALK expression. Mol Cancer. 2010;9:188

21. Li H, Pan Y, Li Y, et al. Frequency of well-identified oncogenic driver mutations in lung adenocarcinoma of smokers varies with histological subtypes and graduated smoking dose. Lung Cancer. 2013;79:8-13.

22. Roberts PJ, Stinchcombe TE, Der CJ, et al. Personalized medicine in non-small-cell lung cancer: is KRAS a useful marker in selecting patients for epidermal growth factor receptortargeted therapy? J Clin Oncol. 2010;28:4769-4777.
23. Wistuba II, Bryant D, Behrens C, et al. Comparison of features of human lung cancer cell lines and their corresponding tumors. Clin Cancer Res. 1999;5:991-1000.

24. Gazdar AF, Girard L, Lockwood WW, et al. Lung cancer cell lines as tools for biomedical discovery and research. J Natl Cancer Inst. 2010;102:1310-1321.

25. Zheng C, Sun YH, Ye XL, et al. Establishment and characterization of primary lung cancer cell lines from Chinese population. Acta Pharmacol Sin. 2011;32:385-392.

26. Yang S, Dong Q, Yao M, et al. Establishment of an experimental human lung adenocarcinoma cell line SPC-A-1BM with high bone metastases potency by $(99 \mathrm{~m}) \mathrm{Tc}-\mathrm{MDP}$ bone scintigraphy. Nucl Med Biol. 2009;36:313-321.

$$
\begin{array}{r}
\text { Address correspondence to: } \\
\text { May Ann Lee, PhD } \\
\text { Cell-Based Assay Development Group } \\
\text { Experimental Therapeutics Centre } \\
31 \text { Biopolis Way, \#03-01 Nanos Building } \\
138669 \\
\text { Singapore }
\end{array}
$$

E-mail: malee@etc.a-star.edu.sg

$\begin{aligned} & \text { Abbreviations Used } \\ \mathrm{ALK} & =\text { anaplastic lymphoma kinase } \\ \mathrm{CGH} & =\text { comparative genomic hybridization } \\ \mathrm{EGFR} & =\text { epidermal growth factor receptor } \\ \mathrm{EMA} & =\text { epithelial membrane antigen } \\ \mathrm{EML} 4 & =\text { echinoderm microtubule-associated } \\ & \quad \text { protein-like } 4 \\ \mathrm{EpCAM} & =\text { epithelial cell adhesion molecule } \\ \mathrm{FBS} & =\text { fetal bovine serum } \\ \mathrm{FISH} & =\text { fluorescence in situ hybridization } \\ \mathrm{HER} 2 & =\text { receptor tyrosine-protein kinase erbB- } 2 \\ \mathrm{IC} & =\text { half maximal inhibitory concentration } \\ \mathrm{KRAS} & =\text { Kirsten rat sarcoma viral oncogene } \\ \mathrm{LOH} & =\text { loss of heterozygosity } \\ \mathrm{NSCLC} & =\text { non }- \text { small cell lung carcinoma } \\ \text { pan-CK } & =\text { pan-cytokeratin } \\ \mathrm{PCR} & =\text { polymerase chain reaction } \\ \mathrm{STR} & =\text { short tandem repeat } \\ \mathrm{TKI} & =\text { tyrosine kinase inhibitor } \\ \mathrm{UGRP} 1 & =\text { uteroglobin-related protein } 1\end{aligned}$

